\title{
Cognitive Deficits in Schizophrenia
}

\author{
Deborah J. Kayman • Marjorie F. Goldstein
}

Published online: 8 February 2012

(C) Springer Science+Business Media, LLC (outside the USA) 2012

\begin{abstract}
Dysfunction in interpersonal relations, work, education, and/or self care is a hallmark of schizophrenia. Numerous studies have attempted to clarify how cognitive deficits may be related to patients' positive and negative symptoms and real-world functioning, and have evaluated interventions to improve patient outcomes. Though effective interventions have proven elusive, the literature continues to grow rapidly. This review of the most recent studies places them in the broader context of the evolution of knowledge of cognition, while focusing on the definition of cognitive domains, the ways in which cognitive deficits may be related to social functioning (among other aspects of real-world functioning), and the patterns and implications of cognitive deficits in old age with respect to patients' health and perceived quality of life. To date, lengthy, multifaceted interventions have shown the most promise. To improve self care, interventions targeting patients' dysfunctional beliefs about aging may be particularly helpful.
\end{abstract}

Keywords Cognitive deficits . Cognitive function . Neurocognition · Social cognition · Social function · Schizophrenia $\cdot$ Aging $\cdot$ Treatment $\cdot$ Remediation $\cdot$ Review

\section{Introduction}

The literature on cognitive deficits in schizophrenia has been growing rapidly since the 1990s [1]. This area of research is important because the effects of cognitive

D. J. Kayman $(\triangle) \cdot$ M. F. Goldstein

VISN 3 Mental Illness Research Education and Clinical Center, James J. Peters Veterans Affairs Medical Center,

130 West Kingsbridge Road,

Bronx, NY 10468, USA

e-mail: Deborah.Kayman@va.gov deficits on patients' functioning and quality of life may persist even when other symptoms remit or are well controlled. Schizophrenia researchers have characterized cognitive deficits, identified neurological changes associated with them, searched for relationships between cognitive domains and specific disabilities, and developed and tested cognitive remediation therapies in hopes of improving patients' realworld functioning and promoting their recovery.

This paper reviews the most recent literature on cognitive deficits in schizophrenia; however, rather than duplicate the reviews and meta-analyses that have been published this year, this paper will place the most recent studies in the broader context of the evolution of knowledge concerning the way in which domains of cognition are defined, how cognitive deficits may be related to performance and realworld functioning (with particular emphasis on social functioning), and the implications of the persistence of cognitive deficits into old age.

\section{Search Methodology}

Electronic databases including PubMed and Ovid were searched, using keywords (e.g., cognitive deficits, neurocognition, social cognition, social function, schizophrenia, aging, treatment, and remediation) and variations of keywords to identify articles published since October 2010. The intent of the authors was to focus on domains of cognition and current knowledge regarding the relationship of these domains to each other and to schizophrenia symptoms and outcomes. For this reason, articles exploring the relationship of cognitive deficits to other phenomena (e.g., blood pressure, obesity, metabolism, abnormal sleep, and antipsychotic medications) were excluded, as were studies of genetics, synaptic dysfunction, neural activity, and structural changes in the brain. Also 
excluded were studies focused primarily on the development and validation of measures.

\section{Defining Characteristics of Schizophrenia}

Defining characteristics of schizophrenia are a) positive and negative symptoms and b) dysfunction in interpersonal relations, work, education, and/or self care. Positive symptoms include reality distortion (e.g., hallucinations or delusions), disorganized speech, and disorganized or catatonic behavior (e.g., motor retardation). Negative symptoms are blunted affect, alogia, asociality, and avolition; anhedonia is an associated feature. Cognitive deficits, formerly included in the category of negative symptoms, are now viewed as a separate domain $[2,3]$.

In schizophrenia, cognitive deficits are extremely common $(75 \%-85 \%$ of patients [4]) and, typically, present a profile that is different from profiles seen in other mental disorders such as dementia, bipolar disorder, and depression [1]. Both retrospective and prospective studies included in a recent review [5] found evidence for the presence of cognitive deficits before the onset of psychotic symptoms. Nonsignificant deficits noted in grade school years reached clinically significant levels in middle and high school. In most studies, cognitive functioning was found to decline most markedly in the period immediately preceding the first episode of psychosis. After diagnosis, cognitive functioning generally stabilizes across time and domains.

As indicated by the studies reviewed below, cognitive deficits appear to predict much of the dysfunction observed in interpersonal relations, work, education, and self care. With reference to behavior, function refers to its purpose or significance. Behavior is dysfunctional when it is abnormal for or inconsistent with its purpose and, as a result, does not fulfill it. Working, maintaining stable residential status, and navigating successfully in various social environments are examples of functional outcome domains that are of great interest to schizophrenia researchers and clinicians.

\section{Domains of Cognition}

Debate is ongoing as to whether symptoms (positive and/or negative) and functional disabilities are independent of or related to each other and, if related, how so. To better identify targets of pharmacological and behavioral intervention and improve measurement of outcomes in clinical trials, domains of cognition were recently standardized by the National Institute of Mental Health in the Measurement and Treatment Research to Improve Cognition in Schizophrenia (MATRICS) Consensus Cognitive Battery (MCCB) $[1,6]$.
Six of the seven cognitive dimensions of the MCCB fall under the heading of neurocognition, which refers to forms of cognition associated with the functioning of one or more specific areas of the brain. The six domains of neurocognition measured by the MCCB are 1) Processing speed, 2) Attention/vigilance, 3) Working memory, 4) Verbal learning, 5) Visual learning, and 6) Reasoning/ Problem-solving. The MATRICS Neurocognition Committee derived these domains from 13 factor analytic studies on cognitive performance [6]. The samples in these studies varied in size (range: 34-209) and composition; though most consisted entirely of people with schizophrenia, some included patients with other serious mental illnesses.

The seventh domain, Social cognition, refers to skills for perception, interpretation, and processing of social information [7]. Skills of social cognition are essential for understanding and benefiting from the social environment. At the time the MCCB was finalized, social cognition was a relatively new area of research, and measurement of attributes and skills related to social cognition had not yet been standardized across research groups [1]. As indicated by the studies reviewed below, this is still the case.

Most recent studies of social cognition have utilized measures for the following domains:

1) Theory of Mind (ToM): Also known as mental state attribution [8•], ToM refers to the ability to make inferences about beliefs, dispositions, and intentions of others. It is thus distinguished from direct perception of the mental states of others.

2) Emotion (perception and) processing: This refers to the ability to identify, facilitate, understand, and manage emotions.

3) Social perception and social knowledge: Social perception refers to the ability to use verbal and nonverbal cues to identify social context, roles (e.g., the nature of the relationships between people), and rules. Social knowledge refers to awareness of a given context and which rules apply in it. Because these are overlapping constructs, they are sometimes combined into one.

4) Attributional bias: This refers to the way an individual typically responds to events; specifically, the kinds of inferences the individual makes about their causes. For example, the tendency to conclude that an action is the result of the actor's hostile intentions is called a hostile attributional bias.

Empathy and self perception are examples of other domains that some investigators (e.g., Lincoln et al. [9]) have chosen to measure. Some investigators also combine domains. For example, Mancuso and colleagues [10 ${ }^{\bullet}$ found support for combining ToM and emotion processing and gave the combination a new label, "inferential and regulatory processes." 
In general, social cognition differs from nonsocial cognition in several ways [7]:

- Neurocognitive stimuli are static and neutral, while by contrast, social stimuli are mutable and have affective import;

- The relationship between the neurocognitive stimulus and the perceiver is unidirectional; that is to say, the perceiver responds to the stimulus but the stimulus does not respond to the perceiver. When the stimulus is social, the relationship between the stimulus and the perceiver is likely to be more complex, more interactive; and

- Neurocognitive measures evaluate performance, while by contrast, social cognition research evaluates not only performance but also biases in reasoning.

\section{Treatments for Schizophrenia: The State of the Art}

\section{Treatments for Positive Symptoms}

Though helpful in reducing positive symptoms, treatment with antipsychotic medications has several important limitations. Patients do not always comply with medication regimes; the symptoms of an estimated $20 \%$ to $30 \%$ of patients do not respond to medication, and of patients who respond initially to medications, a substantial minority experiences relapses [11]. These shortcomings argue for the development and testing not only of new pharmacological agents but also of nonpharmacological interventions for positive symptoms, including interventions that utilize cognitive strategies. One recent example is a metacognitive therapy (MCT) for delusional symptoms [11]. The therapy consists of an eight-session group intervention followed by eight individual sessions with a therapist. A variant of cognitive-behavioral therapy for psychosis, MCT targets cognitive biases, such as jumping to conclusions, which were hypothesized to contribute to the formation and maintenance of delusions. In this randomized clinical trial, patients in the experimental condition $(n=24)$ received MCT; patients in control condition $(n=24)$ received individual cognitive remediation treatment by computer. After treatment, recipients of MCT showed significantly greater decreases in delusion severity than control patients.

\section{Treatments for Negative Symptoms}

Pharmacologic treatment of negative symptoms has had disappointing results to date. Effect sizes in medication trials have ranged from small to nonexistent [12]. Results for nonpharmacological alternatives have been almost as disappointing. Though cognitive remediation therapy has been shown to have an impact on negative symptoms, effect sizes measured after treatment have been small and, at subsequent follow-up, no longer significant $[13 \bullet \bullet]$.

\section{Treatments for Cognitive Deficits}

To date, efficacy in improving cognition has not been established for any pharmacological agent. While cholinesterase inhibitors have shown modest benefits for patients with Alzheimer's disease, trials of these medications in schizophrenia have been less successful. Small placebo-controlled trials of rivastigmine (Exelon [Novartis, East Hanover, NJ]) showed no benefit. Similarly, in a sample of 245 patients, those randomly assigned to receive donepezil (Aricept [Eisai Co., Ltd., Tokyo, Japan and Pfizer, New York, NY]) did no better on cognitive tests than those who took a placebo. Aricept also was associated with a worsening of negative symptoms. Effects of galantamine (Razadyne [Janssen Pharmaceuticals, Inc. \{a pharmaceutical company of Johnson \& Johnson\}, Titusville, NJ]) have been mixed. In a placebo-controlled trial with 86 patients, patients who took Razadyne scored better on some tests and worse on others. No improvement in test scores was seen in a placebo-controlled trial by Johnson \& Johnson with a sample of 100 patients. As in the case of cholinesterase inhibitors, promising findings obtained in small pilot studies with other classes of drugs targeting various biological mechanisms (e.g., dopamine neurotransmission and glutamatergic dysregulation) have not been borne out in larger replication trials [14].

These disappointing findings have stimulated the development and testing of nonpharmacological approaches, such as computer-assisted cognitive remediation. In a recent review by Wykes and colleagues [13••], which will be discussed in greater detail below, 39 such trials were included. Consistent with findings obtained in that review, a more recent randomized clinical trial of computer-assisted cognitive remediation [15] showed that, in this small sample $(n=77)$, performance improved significantly for the experimental group $(n=39)$ on several cognitive domains, but symptoms, social functioning, and quality of life were unchanged.

More promising findings were obtained in another recent randomized clinical trial, in which patients in the experimental group were offered standard treatment plus a manualized experimental intervention consisting of weekly 50min sessions with a therapist for up to 18 months [16•]. The experimental intervention targeted domains of social and neurocognition, as well as dysfunctional beliefs thought to underlie positive and negative symptoms. At follow-up, improvement in global functioning and in positive and negative symptoms was significantly greater for the experimental group $(n=31)$ than for the comparison group $(n=29)$. However, because the study design did not control for the 
extra attention patients in the experimental condition received from their therapists and did not follow patients for a period after their completion of the experimental treatment, further research with larger samples is needed to confirm these findings.

\section{Recent Reviews and Meta-analyses of Clinical Trials of Cognitive Interventions}

As noted above, a recent meta-analysis of findings from clinical trials of cognitive remediation therapies for schizophrenia included 39 reports published before June 2009 [13 • $]$. The total number of participants in these studies was 2104; however, most of the individual studies were small, with a mean sample size of 53 (range: 10-145). Effect sizes measured after treatment were small and, at subsequent follow-up, no longer significant, except for global cognition and functioning. Effects on functioning were significantly stronger when patients received both cognitive remediation therapy and other psychiatric rehabilitation (e.g., socialization groups and art therapy) and stronger still when the cognitive therapy provided to recipients of such rehabilitation included practice drills and strategy training rather than practice drills alone. No effects were found for age (mean: 35.8 years; range: $15.3-48.3$ years).

Another recent article reviewed 36 studies representing 30 years of research on a manualized group intervention for cognition in schizophrenia [17], called Integrated Psychological Therapy (IPT) because it integrates neurocognitive and social cognitive treatments. The studies were diverse in sample size (range: 12-143; mean: 44.5) and methodology (e.g., four studies had no control group). Average treatment length was 16.4 weeks. Improvements in neurocognition, social cognition, psychosocial functioning, and negative symptoms that were achieved in treatment were sustained through the follow-up period, which, on average, was 8.1 months long.

\section{Recent Research on Social Cognition}

As noted above, social cognition is a relatively new area of research; consequently, its measurement is not yet standardized. To date, studies of social cognition have been largely exploratory in nature, many with the goal of further defining social cognition and its relationships to other domains such as neurocognition, positive and negative symptoms, and social functioning, for which some standardized measures are available. Studies conducted to date have only partially attained these goals. Recent cross-sectional studies and a recent review of earlier studies are summarized below.
Fett and colleagues [18] looked for functional outcomes of neuro- and social cognition in a meta-analysis of 52 cross-sectional studies published between 1997 and 2009. They found that social cognition (particularly ToM) was more strongly associated with functional outcomes than neurocognition; however, findings explained only $25 \%$ of the variance, suggesting that factors other than, or in addition to, social cognition influence functional outcomes. This is consistent with the finding of Wykes and colleagues [13••] that even when test performance improves, there may be little to no effect on real-world functioning.

Kosmidis and colleagues [19] compared 28 patients with schizophrenia to 30 healthy control patients, using role play performance to assess social competence. The participants' mean age was 37 years (range: $23-55$ years); 79\% were male. In their analysis, the investigators found relationships between most of the functions they hypothesized to be relevant to ToM and social competence; however, they did not find relationships between neurocognitive variables and social competence.

Lincoln and colleagues [9] compared 75 adults with schizophrenia $(n=66)$ or schizoaffective disorder $(n=9)$ with 75 healthy control patients. The sample included a mix of outpatients $(n=59)$ and inpatients $(n=16)$. The participants' mean age was 34 years; $78 \%$ were male. Domains of social cognition (ToM and self esteem) were found to be associated with negative symptoms, a finding that lent support to the study hypothesis that cognitive deficits may underlie negative symptoms (ie, social withdrawal may be a consequence of negative self perceptions or perceptions of rejection).

Couture and colleagues [20] enrolled 178 participants in a clinical trial. The participants' mean age was 45.9 years; $63.5 \%$ were male; and all were diagnosed with schizophrenia or schizoaffective disorder. Findings from patient self-report data, obtained at baseline, suggested that social cognition (and ToM in particular) is a mediator between neurocognition and functioning. ToM alone explained a small portion of the variance in self-reported functioning.

Mancuso and colleagues [10•] enrolled 85 participants in a clinical trial. The participants' mean age was 48.5 years; $89.4 \%$ were male. All were diagnosed with schizophrenia (68.2\%), schizoaffective disorder $(22.4 \%)$, or unspecified psychosis $(9.4 \%)$. Based on their factor analysis of data obtained at baseline, the investigators proposed three new constructs for dimensions of social cognition: "hostile attributional style," "higher-level inferential and regulatory processes," and "lower-level social cue detection." These three factors explained a total of $54 \%$ of the variance in the impairment of social cognition. 


\section{Studies to Identify Patient Profiles Across Domains of Cognition and/or Symptoms}

A new and noteworthy area of research aims to identify patient profiles, which, with further study, may prove helpful to clinicians by providing a more holistic picture of how the patient experiences the social environment and suggest priority areas for intervention. Recent studies to identify patient profiles are summarized below.

Bell and colleagues $[21 \bullet \bullet]$ recruited and assessed a sample of 77 clinically stable outpatients diagnosed with schizophrenia $(n=52)$ or schizoaffective disorder $(n=25)$. The participants' mean age was 43.4 years; $56 \%$ were male. The investigators found three symptom/deficit clusters that, in their opinion, closely correspond to patterns clinicians see in practice (see Table 1).

Dawes and colleagues [22••] found five distinct neurocognitive clusters or profiles of cognitive strengths and weaknesses in a sample of 144 outpatients with schizophrenia or schizoaffective disorder. Participants' mean age was 51.6 years (range: 40-70 years); 61\% were male and, though no participants were chronically institutionalized (that is, residing in large facilities that separate them from the general population), $63 \%$ resided in board-and-care homes. To identify specific areas of relative strength or weakness, scores for each dimension of neurocognition were compared to the participant's overall score (see Table 2).

A noteworthy finding in this study was that, though Abstraction/Cognitive Flexibility was relatively weak in clusters 2 through 5, a substantial portion of the sample (13\%) fell into cluster 1 , the only cluster that was not weak in this respect. In addition, patients in cluster 5 had higher levels of education and were more likely to be Caucasian, suggesting that some deficits may be affected by environmental factors. Finally, of all the studies summarized above, this one was the only one in which the mean age was over 50 years.

\section{Recent Research on Cognition and Aging in Schizophrenia}

A recent theory-building article [23•] discusses schizophrenia and aging in three dimensions: physical, cognitive, and psychosocial. The authors point to evidence that physical aging is accelerated in people with serious mental illness, including schizophrenia, for reasons that are not yet fully understood. Regarding cognitive aging, the authors highlight findings from recent longitudinal studies on communitydwelling patients, while dismissing studies on cognitive aging in institutionalized patients (that is, those who reside in large facilities, in isolation from the community), because such patients currently constitute only $10 \%$ of the total population with schizophrenia. The rest are "community-dwelling," a label that runs the gamut from patients living independently and visiting a local mental health center as outpatients to patients living in residential facilities such as supportive housing and group homes. Findings from studies on such patients indicate that their rate of cognitive decline is parallel to that of the general population. With respect to psychosocial aging, the authors cite several studies showing that functioning improves with age, for reasons not yet clear. Cognition-enhancing treatments may change the brain (ie, by preserving and/or increasing brain growth). Patients' perceptions concerning their recovery are heterogeneous, ranging from hopelessness and preoccupation with lost opportunities to hopefulness and pleasure over attainment of long-derailed goals.

Unfortunately, the research literature on cognition and aging is sparse [24••]. Two studies and a review article, all published in 2010, are summarized below.

A randomized clinical trial of a program integrating health care and social rehabilitation for community-dwelling patients [25•] followed a sample of 183 patients (mean age: 60 years) for 3 years. In this sample, 157 patients $(86 \%)$ had diagnoses on the schizophrenia spectrum; of these, $77(49 \%)$ were diagnosed with schizophrenia. The intervention was shown to have a high retention rate and to improve psychosocial functioning, with moderate effect sizes in the range of 0.37 to 0.63 . For reasons that remained unexplained, men improved more than women.

A cross-sectional study compared members of the general population $(n=113)$ with community-dwelling individuals diagnosed with schizophrenia $(n=198)$ [26]. The aims of the study were 1) to determine prevalence of successful aging, and 2) to look for associations between variables indicative of a) successful aging in general and b) successful
Table 1 Symptom/deficit clusters [20]

\begin{tabular}{|c|c|c|c|}
\hline \multicolumn{2}{|l|}{ Profile } & \multirow{2}{*}{$\frac{\text { Patients, } \%(n)^{*}}{31(24)}$} & \multirow{2}{*}{$\begin{array}{l}\text { Characteristics } \\
\text { Avoids social interactions generally, so stays } \\
\text { out of trouble }\end{array}$} \\
\hline $\mathrm{HN}$ & High negative symptoms & & \\
\hline LSC & $\begin{array}{l}\text { Low social cognition; Low } \\
\text { negative symptoms }\end{array}$ & $38(26)$ & $\begin{array}{l}\text { Though active, accomplishes little and gets } \\
\text { into trouble }\end{array}$ \\
\hline HSC & $\begin{array}{l}\text { High social cognition; Low } \\
\text { negative symptoms }\end{array}$ & $31(27)$ & $\begin{array}{l}\text { Has better social judgment, functions better } \\
\text { in community, and seeks help more readily }\end{array}$ \\
\hline
\end{tabular}


Table 2 Neurocognitive profiles $[21 \bullet \bullet]$

\begin{tabular}{llll}
\hline Cluster & Patients, $\%(n)^{*}$ & \multicolumn{2}{l}{ Areas of relative strength/weakness } \\
\cline { 3 - 4 } & & Strengths & Weaknesses \\
\hline 1 & $13(19)$ & Visual Learning \& Memory \\
2 & $26(38)$ & Verbal Comprehension; & $\begin{array}{c}\text { Abstraction/Cognitive Flexibility; Auditory } \\
\text { Learning \& Memory; Visual Learning \& } \\
\end{array}$ \\
& $28(40)$ & Processing Speed & Memory \\
3 & $12(17)$ & Abstraction/Cognitive Flexibility \\
4 & $21(30)$ & Visual Learning & $\begin{array}{c}\text { Abstraction/Cognitive Flexibility; Auditory } \\
\text { Learning \& Memory }\end{array}$ \\
& & Verbal Comprehension & Abstraction/Cognitive Flexibility; Visual \\
& & & Learning \& Memory \\
\hline
\end{tabular}

aging in individuals with schizophrenia. The mean age of participants with schizophrenia was 61.5 years and 63 years for those in the comparison group; in both groups, 51\% were male. Participants from the general population were 10 times more likely to meet the investigators' criteria for successful aging than participants with schizophrenia. Lower severity of negative symptoms and higher perceived quality of life were the only variables associated with successful aging in participants with schizophrenia. The authors concluded that prospects for recovery are remote, given the dearth of effective treatments for negative symptoms and the obstacles to intervening to improve quality of life. Confidence in these findings is undermined by problems in the instrument utilized to measure quality of life. The instrument (Quality of Life Index [27]) consists of 32 items on a six-point Likert scale that ask the respondent to indicate satisfaction with, and the importance of, each item on a very diverse and lengthy menu (including, but not limited to, health, marriage, stress, standard of living, education, personal faith, and personal appearance). Some of these items may be more amenable to intervention than others. Although the scoring algorithm adjusts satisfaction responses for the importance responses, the resulting composite score gives no indication as to which components are of greatest weight.

Meesters and colleagues [24••] reviewed 36 cross-sectional and longitudinal studies on social support and social skills among older community-dwelling patients published before May 2009. The following key findings were included:

- Gender and age differences at onset of disease complicate the interpretation of findings from the studies reviewed;

- Overall, older people with schizophrenia are behind their healthy age-peers with respect to social achievements;

- Older people with schizophrenia, like younger people with the same diagnosis, are heterogeneous. They range from one extreme, where they are severely incapacitated and socially isolated, to the other, where they are near normal in cognitive functioning and social integration;
- Positive symptoms and social functioning appear to be unrelated;

- The relationship of specific cognitive domains to specific areas of social functioning is still debated; and

- Little variance in real-world social functioning is explained by social skills measured during testing. Differences in motivation, availability of social support, and environmental stressors may be responsible for disparities between performance in simulated settings and the actual occurrence of behaviors in community.

\section{Conclusions}

Though cognitive deficits in schizophrenia have proven difficult to assess and treat, it is important to continue to study them because they are so prevalent and so damaging to patients' quality of life. The interventions that have shown the most promise to date are those that are multifaceted and lengthy. Why their results were better than for brief interventions with a narrower focus is still unknown. Perhaps the difference is due to a yet to be identified feature of one or more of the components of the intervention. Another possibility is simply that the patients receive attention for a longer period. More research is needed to resolve these questions. Given that many brief neurocognitive interventions already have been tested, with disappointing results, trials comparing more ambitious interventions may be a better investment despite the added cost.

A recent prospective study collected data at baseline from 83 middle-aged and elderly patients with schizophrenia (mean age: 58.5 years; range: 41-79 years) about their positive and negative self perceptions of aging, and followed the sample for 3 months, recording all acute medical events that occurred in that period [28•]. Findings supported the investigators' hypothesis that negative beliefs about aging would predict the patients' physical health status. Patients with schizophrenia whose views of their own aging 
are negative may be less inclined than patients with more positive views to seek medical help or engage in self care. Though cognitive interventions targeting negative beliefs about aging may be particularly helpful for this group, intervention trials to date all have targeted other dysfunctional beliefs. Perhaps future research will help to close this gap and, in this and other ways, promote recovery for older persons with schizophrenia.

Acknowledgements This review was supported with resources of the Mental Illness Research Education and Clinical Center in Veterans Integrated Service Network 3, and facilities of the James J. Peters VA Medical Center, Bronx, NY.

Disclosures No potential conflicts of interest relevant to this article were reported.

\section{References}

Papers of particular interest, published recently, have been highlighted as follows:

- Of importance

•- Of major importance

1. Green MF, Nuechterlein KH, Gold JM, et al. Approaching a consensus cognitive battery for clinical trials in schizophrenia: the NIMH-MATRICS conference to select cognitive domains and test criteria. Biol Psychiatry. 2004;56(5):301-7.

2. American Psychiatric Association: Diagnostic and statistical manual of mental disorders. 4. ed., text rev., 5th printing. ed. Washington DC: APA; 2005.

3. Kirkpatrick B. The NIMH-MATRICS Consensus Statement on Negative Symptoms. Schizophr Bull. 2006;32(2):214-9.

4. Reichenberg A, Weiser M, Caspi A, et al. Premorbid intellectual functioning and risk of schizophrenia and spectrum disorders. J Clin Exp Neuropsychol. 2006;28(2):193-207.

5. Lewandowski KE, Cohen BM, Ongur D. Evolution of neuropsychological dysfunction during the course of schizophrenia and bipolar disorder. Psychol Med. 2011;41(2):225-41.

6. Nuechterlein KH, Barch DM, Gold JM, et al. Identification of separable cognitive factors in schizophrenia. Schizophr Res. 2004;72(1):29-39.

7. Penn DL, Corrigan PW, Bentall RP, et al. Social cognition in schizophrenia. Psychol Bull. 1997;121:114-32.

8. - Green MF, Penn DL, Bentall R, et al.: Social Cognition in Schizophrenia: An NIMH Workshop on Definitions, Assessment, and Research Opportunities. Schizophr Bull 2008, 34:1211-20. This article lists domains of social cognition and provides clear definitions for them.

9. Lincoln TM, Mehl S, Kesting M-L, Rief W. Negative symptoms and social cognition: identifying targets for psychological interventions. Schizophr Bull. 2011;37 Suppl 2:S23-32.

10. • Mancuso F, Horan WP, Kern RS, Green MF: Social cognition in psychosis: multidimensional structure, clinical correlates, and relationship with functional outcome. Schizophr Res 2011 Feb;125 (2-3):143-51. This study, the first to perform factor analysis on social cognitive domains, identified three factors that explained $54 \%$ of the variance in impairment. The authors analyzed baseline data from a randomized clinical trial.
11. Moritz S, Veckenstedt R, Randjbar S, et al. Antipsychotic treatment beyond antipsychotics: metacognitive intervention for schizophrenia patients improves delusional symptoms. Psychol Med. 2011;41(9):1823-32.

12. Mäkinen J, Miettunen J, Isohanni M, Koponen H. Negative symptoms in schizophrenia: a review. Nord J Psychiatry. 2008;62 (5):334-41.

13. •- Wykes T, Huddy V, Cellard C, et al.: A meta-analysis of cognitive remediation for schizophrenia: methodology and effect sizes. Am J Psychiatry 2011, 168(5):472-85. Findings from this meta-analysis of clinical trial findings published before June 2009 have been borne out in more recent studies. A particularly important finding is that effects on functioning were stronger when patients received multifaceted interventions including strategy training.

14. Goff DC, Hill M, Barch D. The treatment of cognitive impairment in schizophrenia. Pharmacol Biochem Behav. 2011;99(2): $245-53$

15. d'Amato T, Bation R, Cochet A, et al. A randomized, controlled trial of computer-assisted cognitive remediation for schizophrenia. Schizophr Res. 2011;125(2-3):284-90.

16. - Grant PM, Huh GA, Perivoliotis D, et al.: Randomized Trial to Evaluate the Efficacy of Cognitive Therapy for Low-Functioning Patients With Schizophrenia. Arch Gen Psychiatry 2011, In Press. The authors report findings from a trial of an 18-month intervention combining group and individual cognitive therapy. Improvement in global functioning and reduction in negative symptoms were significantly greater for the experimental group than for the comparison group, which received medication and psychiatric rehabilitation. These promising findings warrant further study.

17. Roder V, Mueller DR, Schmidt SJ. Effectiveness of integrated psychological therapy (IPT) for schizophrenia patients: a research update. Schizophr Bull. 2011;37 Suppl 2:S71-9.

18. Fett A-KJ, Viechtbauer W, Dominguez M-de-G, et al. The relationship between neurocognition and social cognition with functional outcomes in schizophrenia: a meta-analysis. Neurosci Biobehav Rev. 2011;35(3):573-88.

19. Kosmidis MH, Giannakou M, Garyfallos G, et al. The Impact of Impaired "Theory of Mind" on Social Interactions in Schizophrenia. J Int Neuropsychol Soc. 2011;17:511-21.

20. Couture SM, Granholm EL, Fish SC. A path model investigation of neurocognition, theory of mind, social competence, negative symptoms and real-world functioning in schizophrenia. Schizophr Res. 2011;125(2-3):152-60.

21. •- Bell MD, Corbera S, Johannesen JK, et al.: Social Cognitive Impairments and Negative Symptoms in Schizophrenia: Are There Subtypes With Distinct Functional Correlates? Schizophr Bull 2011, In Press. Data analysis from this cross-sectional study identified three symptom/cognitive deficit clusters that correspond to patterns clinicians see in practice.

22. • Dawes SE, Jeste DV, Palmer BW: Cognitive profiles in persons with chronic schizophrenia. J Clin Exp Neuropsychol 2011, 33 (8):929-36. Data analysis identified five distinct clusters of cognitive strengths and weaknesses.

23. - Jeste DV, Wolkowitz OM, Palmer BW: Divergent trajectories of physical, cognitive, and psychosocial aging in schizophrenia. Schizophr Bull 2011, 37(3):451-5. The authors discuss schizophrenia and aging in three dimensions: physical, cognitive, and psychosocial.

24. •• Meesters PD, Stek ML, Comijs HC, et al.: Social Functioning Among Older Community-Dwelling Patients With Schizophrenia: A Review. Am J Geriatr Psychiatry 2010, 18:862-78. All 36 crosssectional and longitudinal studies in this review focus on social support and social skills among aging patients with schizophrenia.

25. - Mueser KT, Pratt SI, Bartels SJ, et al.: Randomized trial of social rehabilitation and integrated health care for older people 
with severe mental illness. J Consult Clin Psychol 2010, 78:561-73. This study compared standard treatment (e.g., medication, individual therapy, and psychiatric rehabilitation) with a manualized, recovery-oriented intervention featuring skills training and patient/provider collaboration on health management. The intervention had a high retention rate and improved psychosocial functioning.

26. Ibrahim F, Cohen CI, Ramirez PM. Successful Aging in Older Adults With Schizophrenia: Prevalence and Associated Factors. Am J Geriatr Psychiatry. 2010;18:879-86.
27. Ferrans CE, Powers MJ. Quality of life index: development and psychometric properties. ANS Adv Nurs Sci. 1985;8(1):15-24.

28. - Cheng S-T, Yip LCY, Jim OTT, Hui ANN: Self-perception of aging and acute medical events in chronically institutionalized middle-aged and older persons with schizophrenia. Int J Geriatr Psychiatry 2011, In Press. In this prospective study, negative beliefs about aging predicted the patients' physical health status. This suggests that cognitive interventions targeting negative beliefs might improve patients' health and quality of life by increasing self care and timely help-seeking. 